\title{
Indications for Rituximab Use in an Integrated Health Care Delivery System
}

\author{
Thomas Delate, PhD, MS; Margaret L. Hansen, PharmD; \\ Amarylis C. Gutierrez, PharmD; and Kim N. Le, PharmD
}

\begin{abstract}
BACKGROUND: Rituximab is a top-selling biologic that was first approved by the FDA in 1997 for a non-Hodgkin lymphoma orphan indication. It has since been approved for additional orphan indications, with rheumatoid arthritis as the only FDA-approved, nonorphan indication. Evidence suggests that rituximab is frequently used off-label, but information on its use over time and indications for use in the United States is limited.

OBJECTIVE: To assess incident rituximab use over time in an integrated health care delivery system.

METHODS: This was a cross-sectional, retrospective study. Data were collected from administrative databases and manual chart reviews. Patients who received their first rituximab infusion between 0ctober 1, 2009, and December 31, 2017, and who were not a part of a clinical trial were included. Indication for use (FDA-approved orphan/nonorphan, off-label) was determined. Proportions of use were assessed over time. Multivariable logistic regression modeling was performed to assess factors associated with receiving rituximab for an FDA-approved indication.

RESULTS: A total of 1,674 patients were included. The majority $(66.4 \%)$ of patients had an FDA-approved indication, with lymphoma being the most common approved indication (66.4\%). The most common indication for off-label use was neurologic conditions (72.7\%), predominantly demyelinating diseases. Off-label indication use increased from $1.2 \%$ in 2009 to $55.6 \%$ in 2017. Factors associated with rituximab use for an FDA-approved indication included increased age (adjusted odds ratio $[A 0 R]=1.05,95 \%$ $\mathrm{Cl}=1.04-1.07$ ) and increased burden of chronic disease (chronic disease score: $A 0 R=1.07,95 \% \mathrm{Cl}=1.02-1.12$; Charlson Comorbidity Index score: $\mathrm{AOR}=3.52,95 \% \mathrm{Cl}=3.03-4.10)$.

CONCLUSIONS: Off-label use of rituximab grew dramatically over the course of the study. With the recent FDA approval of the rituximab biosimilar and its expected lower price, off-label use will likely continue to rise. Opportunities for cost savings and to ensure appropriate use of these medications should be evaluated.
\end{abstract}

J Manag Care Spec Pharm. 2020;26(7):832-38

Copyright $\odot 2020$, Academy of Managed Care Pharmacy. All rights reserved.

\section{What is already known about this subject}

Rituximab is a top-selling biologic that has FDA-approved orphan and nonorphan indications.

Evidence suggests that rituximab is used for both FDA-approved and off-label indications, but information on its use in the United States is limited.

\section{What this study adds}

Overall, the majority of patients had an FDA-approved indication, with lymphoma being the most common approved indication. The percentage of patients receiving rituximab for an off-label indication increased from $1.2 \%$ in 2009 to $55.6 \%$ in 2017.

The most common indication for off-label use was neurologic conditions, predominantly demyelinating diseases.

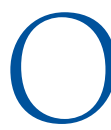
rphan drugs are medications designed to treat rare indications that affect $<200,000$ patients or $>200,000$ patients if it is not expected that the costs of developing and marketing the treatment drug will be recovered. ${ }^{1}$ Historically, pharmaceutical companies have been reluctant to develop orphan drugs because of the low probability of recovering their investment. ${ }^{2}$ In 1983, the U.S. Orphan Drug Act was passed to provide economic incentives to develop orphan drugs. ${ }^{1}$ This act has been amended so that researchers can use grants specifically for developing orphan drugs. ${ }^{3}$ Additionally, firms can receive tax credits for expenditures incurred during clinical testing, waive prescription drug user fees, and have 7 years of market exclusivity (i.e., no other medication will receive approval for the same indication unless clinical superiority is demonstrated) for each FDA-designated orphan drug indication. ${ }^{3}$

Orphan drugs have approval from the U.S. Food and Drug Administration (FDA) to be marketed for an orphan indication; orphan-designated drugs are in the process of obtaining FDA approval to be marketed as an orphan drug; and orphan drugs can have FDA approval for nonorphan indications. ${ }^{4}$ Despite the financial incentives that manufacturers receive for developing drugs that have orphan indications, these medications are often very expensive. In 2017, the average cost per patient per year for an orphan drug was $\$ 147,308$, compared with $\$ 30,708$ for a nonorphan specialty drug. ${ }^{5}$ The annual revenue from orphan drugs suggests that they have a high cost per unit and/or there is widespread use outside of the FDA-approved orphan indications. ${ }^{6}$

Rituximab is an anti-CD20 monoclonal antibody administered as an intravenous infusion. It was first approved in the United States in 1997 for the orphan indication of non-Hodgkin lymphoma. ${ }^{7}$ It has since been approved for 4 additional orphan 
indications: chronic lymphocytic leukemia (2010), Wegener granulomatosis (2011), microscopic polyangiitis (2011), and pemphigus vulgaris (2018). ${ }^{7}$ Additionally, rituximab has orphan designations for immune thrombocytopenic purpura, eosinophilic granulomatosis with polyangiitis (Churg-Strauss syndrome), and Rasmussen encephalitis. ${ }^{7}$ Rheumatoid arthritis is the only FDA-approved, nonorphan indication for rituximab. ${ }^{7}$ Rituximab is considered a blockbuster specialty drug with nearly $\$ 4.2$ billion in U.S. sales in $2017 .^{5}$ Nevertheless, the peer-reviewed literature on orphan-related rituximab use is limited. ${ }^{8-10}$ Thus, the purpose of this study was to provide a contemporary assessment of rituximab and its indications for use over time to illustrate the paths orphan drugs can take during their product life cycles.

\section{Methods}

\section{Study Design and Setting}

This was a retrospective, cross-sectional study conducted at Kaiser Permanente Colorado (KPCO), an integrated health care delivery system that provides care to over 630,000 members in Colorado. Patients who received their first (index) infusion of rituximab between October 1, 2009, and December 31, 2017, were included in the study. Patients were categorized as receiving their first rituximab infusion for an "approved indication" if the diagnosis from the index infusion encounter was for an FDA-approved orphan or nonorphan indication or an orphandesignated (pemphigus vulgaris) indication as of the infusion date (Table 1). While pemphigus vulgaris was an orphandesignated indication during the study period, it received FDAapproved orphan drug status after the study period. ${ }^{7}$ Thus, it was included with FDA-approved orphan and nonorphan indications for this study. Patients not receiving rituximab for one of the approved indications were categorized as "off-label" use. Indication of use for the orphan drug was assessed during the 365 days before and 90 days after the index infusion.

KPCO owns and operates outpatient infusion centers and uses an electronic health record system where detailed information about infusions (e.g., infused medication ordered and associated indication) is captured electronically. In addition, coded and free-text medical, pharmacy, emergency department, hospitalization, expenditure, and membership information from within the delivery system, as well as from other contracted and affiliated facilities, are captured in KPCO's administrative electronic health record and claims databases. All aspects of this study were reviewed and approved by the KPCO Institutional Review Board.

\section{Patient Population}

Patients who received an infusion of rituximab between October 1, 2009, and December 31, 2017, had no other rituximab infusion recorded during the previous 365 days, and had been KPCO members for a minimum of 90 days before the index infusion were included. Patients who received rituximab as part of a clinical trial were excluded.
TABLE 1 FDA-Approved Rituximab Indications ${ }^{7}$

\begin{tabular}{l|c|c} 
Indication & $\begin{array}{c}\text { FDA } \\
\text { Approval Date }\end{array}$ & $\begin{array}{c}\text { Orphan } \\
\text { Designation }\end{array}$ \\
\hline Non-Hodgkin B-cell lymphoma & $11 / 26 / 1997$ & Yes \\
\hline Wegener granulomatosis & $4 / 19 / 2011$ & Yes \\
\hline Microscopic polyangiitis & $4 / 19 / 2011$ & Yes \\
\hline Chronic lymphocytic leukemia & $6 / 22 / 2017$ & Yes \\
\hline Pemphigus vulgaris & $6 / 7 / 2018$ & Yes \\
\hline Immune thrombocytopenia purpura & - & Yes \\
\hline Churg-Strauss syndrome & - & Yes \\
\hline Rasmussen encephalitis & - & Yes \\
\hline Rheumatoid arthritis & $2 / 28 / 2006$ & No \\
\hline
\end{tabular}

FDA = U.S. Food and Drug Administration.

\section{Study Outcomes}

The primary study outcome was the proportion of patients with an approved indication during the study period. The secondary outcomes included a comparison of the characteristics between patients with and without an approved indication, description of the indications, and the annual proportions of FDA-approved orphan/orphan designation, FDA-approved nonorphan indications, or off-label indications during the study period. In addition, factors associated with receiving rituximab for an approved indication were assessed.

\section{Data Collection}

A detailed list of International Classification of Disease, Ninth/ Tenth Revision, Clinical Modification (ICD-9-CM, ICD-10-CM) codes for FDA-approved orphan/orphan designation and nonorphan diagnoses was compiled with input from subject matter experts (codes available upon request). Electronic medical record and claims databases were queried with the codes to identify the indication associated with the rituximab infusion. In addition, International Classification of Diseases for Oncology, Third Revision (ICD-O) and morphology codes were used to query the KPCO tumor registry for approved, cancer-related indications diagnosed before the index date.

For patients without an indication linked to the rituximab infusion or a cancer-related indication, administrative databases were queried first for ICD-9-CM or ICD-10-CM codes of approved indications for rituximab during the 365 days before or 90 days after the index date. For patients without an ICD-9-CM or ICD-10-CM code for an approved rituximab indication, administrative databases were queried for ICD9-CM and ICD-10-CM codes for common off-label indications during the 12 months before and 90 days after the index date. Finally, manual chart reviews were conducted to determine the indication for any remaining patients. Given the variability of associated off-label indications, these indications were categorized based on the affected physiologic system or class of disorder. Samples of data extracted from the administrative 
Indications for Rituximab Use in an Integrated Health Care Delivery System

TABLE 2 Characteristics of Patients with a Rituximab Infusion by Indication Approval Status (N=1,674)

\begin{tabular}{|c|c|c|c|c|c|}
\hline \multirow{2}{*}{$\begin{array}{l}\text { Characteristic } \\
\text { Mean age (SD) }\end{array}$} & \multicolumn{2}{|c|}{$\begin{array}{l}\text { Approved Indication } \\
(\mathrm{n}=1,111)\end{array}$} & \multicolumn{2}{|c|}{$\begin{array}{l}\text { Off-Label Indication } \\
\qquad(\mathrm{n}=563)\end{array}$} & \multirow{2}{*}{$\frac{P \text { Value }}{<0.001}$} \\
\hline & 64.4 & $(14.0)$ & 48.0 & (14.3) & \\
\hline Female, n (\%) & 565 & $(50.9)$ & 200 & $(35.5)$ & $<0.001$ \\
\hline \multicolumn{5}{|l|}{ Race, n (\%) } & $<0.001$ \\
\hline White & 885 & $(79.7)$ & 406 & $(72.1)$ & \\
\hline Other & 109 & $(9.8)$ & 95 & $(16.9)$ & \\
\hline Unknown/undeclared & 117 & $(10.5)$ & 62 & $(11.0)$ & \\
\hline Hispanic ethnicity, n (\%) & 119 & $(10.7)$ & 79 & $(14.0)$ & 0.047 \\
\hline \multicolumn{6}{|l|}{ Comorbidities, $\mathrm{n}(\%)^{\mathrm{b}}$} \\
\hline Chronic pulmonary disease & 266 & $(23.9)$ & 85 & $(15.1)$ & $<0.001$ \\
\hline Congestive heart failure & 84 & $(7.6)$ & 17 & $(3.0)$ & 0.002 \\
\hline Diabetes & 183 & $(16.5)$ & 55 & (9.8) & 0.002 \\
\hline Liver disease & 106 & $(9.5)$ & 28 & $(5.0)$ & 0.001 \\
\hline Malignancy & 842 & $(75.9)$ & 31 & (5.5) & $<0.001$ \\
\hline Renal disease & 208 & $(18.7)$ & 74 & $(13.1)$ & 0.004 \\
\hline Rheumatologic disease & 172 & $(15.5)$ & 36 & $(6.4)$ & $<0.001$ \\
\hline Mean Charlson Comorbidity Index score (SD) & 3.4 & $(2.5)$ & 1.0 & $(1.7)$ & $<0.001$ \\
\hline Mean chronic disease score (SD) & 5.1 & $(3.8)$ & 3.4 & (3.6) & $<0.001$ \\
\hline \multicolumn{6}{|l|}{ Benefit design, $n(\%)$} \\
\hline Medicaid beneficiary & 38 & (3.4) & 39 & $(6.9)$ & 0.001 \\
\hline Medicare beneficiary & 653 & $(58.8)$ & 142 & $(25.2)$ & $<0.001$ \\
\hline High deductible health plan & 43 & $(3.9)$ & 41 & $(7.3)$ & 0.003 \\
\hline
\end{tabular}

a As of index infusion date.

bDiagnosed during the 6 months before first infusion date.

$S D=$ standard deviation.

databases were chart reviewed manually for accuracy. In cases where multiple diagnosis codes were associated with the order, the patient was categorized in a hierarchically manner: FDAapproved orphan/orphan-designation, FDA-approved nonorphan, and then off-label.

Patient characteristics and outcomes were identified through queries of KPCO's administrative databases. Patient benefit designations were collected as of the index infusion date. Comorbidities and medication dispensing dates and codes were collected during the 6 months before the index date.

\section{Data Analysis}

This was a naturalistic, observational study, so no a priori sample size or power calculations were performed. Therefore, all patients meeting the inclusion and exclusion criteria were included. Age was calculated as of the index date. Approved indications were categorized as autoimmune, chronic lymphocytic leukemia, non-Hodgkin lymphoma, and rheumatologic diseases. Off-label indications were categorized as autoimmune, end-organ manifestation, hematologic, neurologic, oncologic, transplant, vascular/dermatologic, and other diseases. A chronic disease score, a validated measure that indicates a patient's burden of chronic illness, was calculated for each patient. ${ }^{11}$ Chronic disease scores range from 0 to 36, with higher values indicating a greater burden of chronic disease. The presence of specific comorbidities was determined using the Quan adaptation of the Charlson Comorbidity Index to give a 30-point comorbidity score. ${ }^{12}$

The proportion of patients with an FDA-approved orphan/ orphan designation, FDA-approved nonorphan, or off-label indication was calculated by summing all patients during the study period/year who were assigned to each category and then dividing this count by all the patients included during the study period/year. This value is reported as a percentage and graphed by study year. A similar process was used to determine the proportion of approved versus nonapproved indications.

Patient characteristics were summarized as means ( \pm standard deviation) for continuous variables and percentages for categorical variables. Characteristics were compared between groups with chi-square tests of association and t-tests or nonparametric equivalents (depending on data distributions) for nominal- and ordinal-level and interval-level data, respectively. A multivariable logistic regression model was constructed to assess factors associated with an approved indication with all patient characteristics except malignancy and rheumatologic disease. These comorbidities, obtained from the Charlson Comorbidity Index, include FDA-approved indications for rituximab, and their inclusion would likely have overfit the model. The alpha was set at 0.05 , and analysis was performed with SAS version 9.4 (SAS Institute, Cary, NC). 


\begin{tabular}{|c|c|c|}
\hline Indication & \multicolumn{2}{|c|}{ Counts, n (\%) } \\
\hline Approved indications & 1,111 & $(66.4)$ \\
\hline Autoimmune $^{\mathrm{a}}$ & 163 & $(14.7)$ \\
\hline Chronic lymphocytic leukemia & 84 & (7.6) \\
\hline Non-Hodgkin lymphoma & 738 & $(66.4)$ \\
\hline Rheumatologic disease & 126 & (11.3) \\
\hline Off-label indications & 563 & $(34.6)$ \\
\hline Autoimmune & 26 & $(4.6)$ \\
\hline End organ manifestation & 22 & $(3.9)$ \\
\hline Hematologic & 41 & (7.3) \\
\hline Neurologic ${ }^{b}$ & 409 & $(72.7)$ \\
\hline Oncologic & 11 & $(2.0)$ \\
\hline Transplant & 16 & $(2.8)$ \\
\hline Vascular/dermatologic & 23 & $(4.1)$ \\
\hline Other & 15 & $(2.7)$ \\
\hline \multicolumn{3}{|c|}{$\begin{array}{l}\text { ancludes Wegener granulomatosis, Churg-Strauss syndrome, microscopic } \\
\text { polyangiitis, immune thrombocytopenic purpura, and pemphigus vulgaris. } \\
\text { b374 (91.4\%) were for demyelinating diseases (including demyelinating } \\
\text { neuropathies, demyelinating central nervous system diseases, and multiple } \\
\text { sclerosis), and } 35 \text { (8.6\%) were for neurologic disorders (including cerebral } \\
\text { ataxia, encephalitis, encephalomyelitis, myasthenia gravis, myoneural disorders, } \\
\text { neuropathies, and stiff-person syndrome). }\end{array}$} \\
\hline
\end{tabular}

\section{Results}

A total of 1,674 patients were included. Of these, 1,111 (66.4\%) and $563(33.6 \%)$ received rituximab for an approved and offlabel indication, respectively (Table 2). In univariate analyses, patients who received rituximab for an off-label indication were younger, more likely to be male, nonwhite, Hispanic, and a Medicaid beneficiary and had a lower burden of chronic disease (all $P<0.05$ ), while patients who received rituximab for an approved indication were more likely to have a malignancy comorbidity and be a Medicare beneficiary (both $P<0.001$ ).

Oncology indications made up approximately three quarters of the approved indications (Table 3). The majority of approved indications were for non-Hodgkin lymphoma (66.4\%) with autoimmune diseases, which include orphan-approved and orphan-designated indications, the next most common indication (14.7\%). Infusions for rheumatoid arthritis, the only approved nonorphan indication, accounted for $11.3 \%$ of the approved indications. The majority of off-label indications were for neurologic diseases $(72.7 \%)$, with hematologic diseases (7.3\%) the next most common indication. The vast majority of neurologic indications were for demyelinating diseases (91.4\%).

While approximately two thirds of all rituximab infusions over the course of the study were for approved indications, use of rituximab for off-label indications increased over time (Figure 1). In 2009, off-label rituximab use comprised 1.2\% of the infusions, but by the end of the study in 2017, off-label use made up over one half of infusions (55.6\%). The use of rituximab for off-label indications increased nearly every year of the study. Conversely, the use for approved orphan indications decreased from 92.8\% of infusions in 2009 to $38.8 \%$ in 2017. The use for approved nonorphan indications ranged from a high of $14.1 \%$ in 2011 to a low of $5.1 \%$ in 2015 .

Patient age, sex, race, Hispanic ethnicity, chronic disease score, Charlson Comorbidity Index score, comorbidities, and benefit design factors were entered in a multivariable logistic regression model to identify factors associated with use for an approved indication (Table 4). Increased age (adjusted odds ratio $[\mathrm{AOR}]=1.05,95 \%$ confidence interval $[\mathrm{CI}]=1.04-1.07)$ and increased burden of chronic disease (chronic disease score: $\mathrm{AOR}=1.07,95 \% \mathrm{CI}=1.02-1.12$; Charlson Comorbidity Index score $=[\mathrm{AOR}=3.52,95 \% \mathrm{CI}=3.03-4.10)$ were associated independently with a higher likelihood of use for an approved indication. Comorbidities (chronic pulmonary disease: $\mathrm{AOR}=0.22$, 95\% CI $=0.10-0.33$; congestive heart failure: $\mathrm{AOR}=0.22,95 \%$ $\mathrm{CI}=0.14-0.33$; diabetes: $\mathrm{AOR}=0.17,95 \% \mathrm{CI}=0.10-0.28$; liver disease: $\mathrm{AOR}=0.31,95 \% \mathrm{CI}=0.17-0.55$; renal insufficiency: $\mathrm{AOR}=0.03,95 \% \mathrm{CI}=0.02-0.05)$ were associated independently with a lower likelihood of use for an approved indication.

\section{Discussion}

This retrospective, cross-sectional study of over 1,600 patients who received a rituximab infusion between 2009 and 2017 identified that most patients received rituximab for an approved indication. Overall, rituximab was used for an approved indication $66.4 \%$ of the time. On the other hand, the proportion of patients who received rituximab for an off-label indication increased by over $4,600 \%$ during the course of the study. The increased use of rituximab for off-label indications began to rise precipitously in the latter half of the study period. By 2017, off-label indications were the most common indications. These findings are important to health plan policymakers, since the launch of lower-cost rituximab biosimilars could lead to further increased use of the biosimilars for label and off-label indications. ${ }^{13,14}$ While rituximab can be a highly effective therapy, especially for diseases that have limited treatment options, it can come at great expense to the patient and health care system when not used as intended.

Few studies have assessed off-label use of rituximab. In the United States, a 2016 data brief (not peer reviewed) was issued by America's Health Insurance Plans (AHIP). The report stated that rituximab was used off-label $20.8 \%$ of the time between 2012 to $2014 . .^{15}$ In our study, off-label use was numerically higher at 28.5\% of patients during 2012-2014. Because the AHIP report relied solely on diagnosis codes in claims data without the ability to link to indications from the infusion or manually review to validate indications, the researchers may have underestimated off-label use. A small, older study of indications for rituximab use at a single academic center in the United States between 1998 and 2001 identified that 75\% of infusions were for off-label indications and that off-label use increased nearly exponentially in comparison with use for 


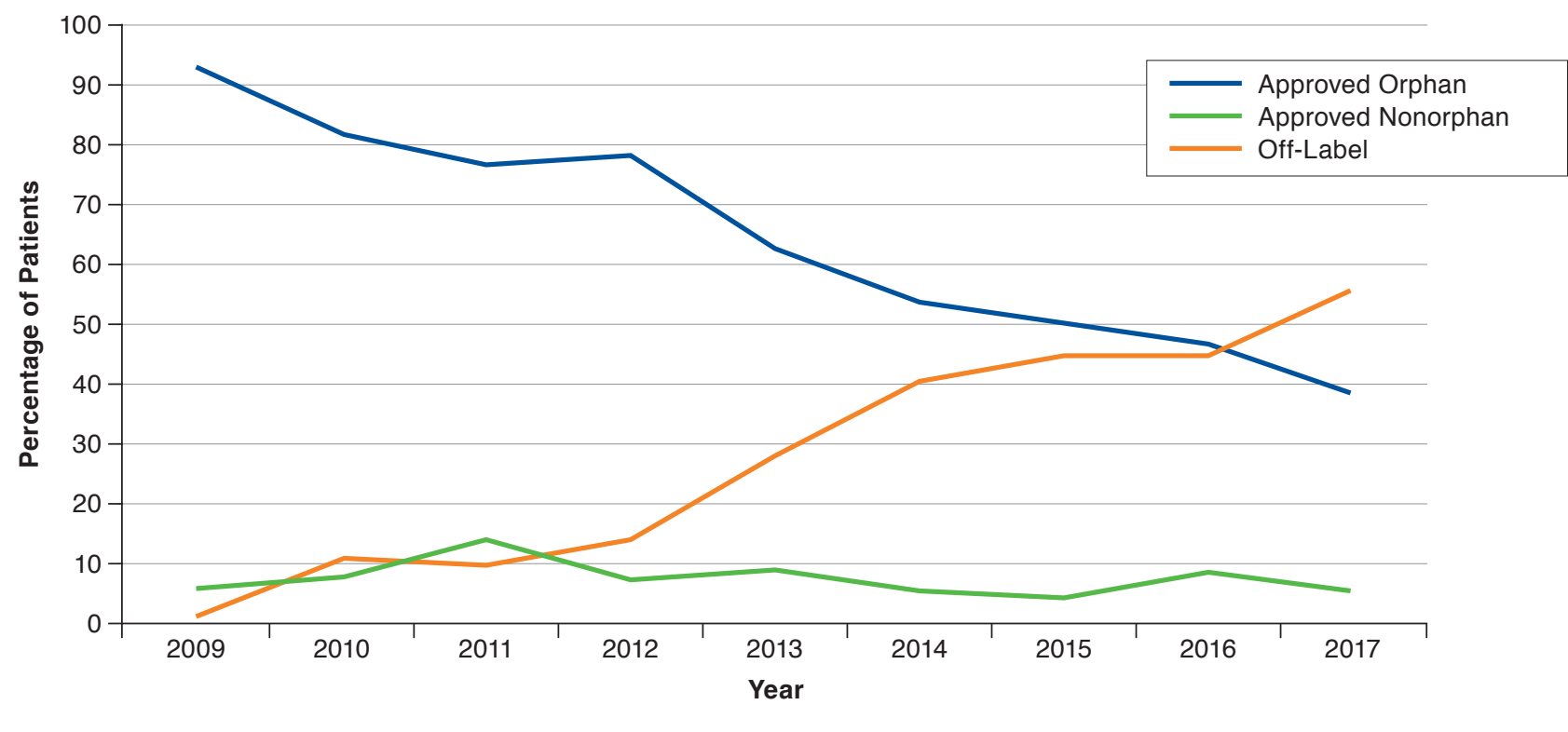

FDA-approved indications. ${ }^{8}$ Van Allen et al. (2011) performed a U.S. commercial insurance database descriptive study of offlabel rituximab use between 2001 and 2007. ${ }^{10}$ They identified 2,782 patients who received rituximab, with $25.3 \%$ receiving rituximab for an off-label indication. ${ }^{10}$ This study was limited to the use of claims data so may have overestimated off-label use. Finally, in a study from Spain, rituximab was the most frequent medication administered for an off-label indication in 1 hospital. ${ }^{16}$ It was administered for 22 different off-label indications, most commonly for transplant-related, dermatologic, autoimmune tissue, and renal indications. ${ }^{16}$ In a retrospective study that evaluated the extent of approved and unapproved uses of 4 nonrituximab orphan drugs, Kesselheim et al. (2012) reported that lidocaine patch and modafinil exhibited a dramatic increase in the proportion of use for off-label indications from 1999 to $2005 .{ }^{6}$ In total, these studies, along with our study, provide evidence of the rapid increase in off-label use and the potential for off-label use to increase.

Treatment of demyelinating neurologic conditions was the primary driver of off-label use in our study. A recent assessment of the Swedish multiple sclerosis (MS) registry identified that rituximab is the most commonly prescribed diseasemodifying therapy for patients with MS. ${ }^{17}$ The uptick in use of rituximab in patients with demyelinating conditions appears to have hinged on the publication of several small studies that evaluated the efficacy and, primarily, safety of B-cell depletion in MS. ${ }^{18-21}$ These studies identified that rituximab reduced inflammatory brain lesions, but they did not assess long-term safety. Also, prescriber comfort with off-label orphan drug use likely contributed to the increased use of rituximab for off-label neurologic use. ${ }^{22}$

We identified that increased age and higher, overall burden of chronic disease were associated with receiving rituximab for an FDA-approved indication, while individual comorbidities (i.e., chronic pulmonary disease, congestive heart failure, liver disease, and renal insufficiency) were associated with receiving rituximab for off-label use. Given that the most common FDA-approved indications are for oncology diagnoses, it would be expected that the patient population would be older and have a greater chronic disease burden. Conversely, off-label use would be expected in diseases that present earlier in life and may affect patients who are otherwise healthy (e.g., neurologic and autoimmune conditions).

\section{Limitations}

While this large study of indications associated with the use of rituximab over time identified a substantial increase in rituximab off-label use, it has limitations to consider when interpreting the results. Despite its large sample size, it was performed within 1 integrated health care delivery system. Other systems may experience differing rates of off-label use. Also, internal prescribing guidelines (e.g., a 2014 KPCO Neurology Department guideline included an option for rituximab therapy in patients with MS) may have inflated off-label use. Not all patients had an indication linked to their infusion. In these cases, we used ICD-9, ICD-10, and ICD-O codes to query administrative data along with manual chart reviews to identify indications. We only assessed patients' first rituximab 


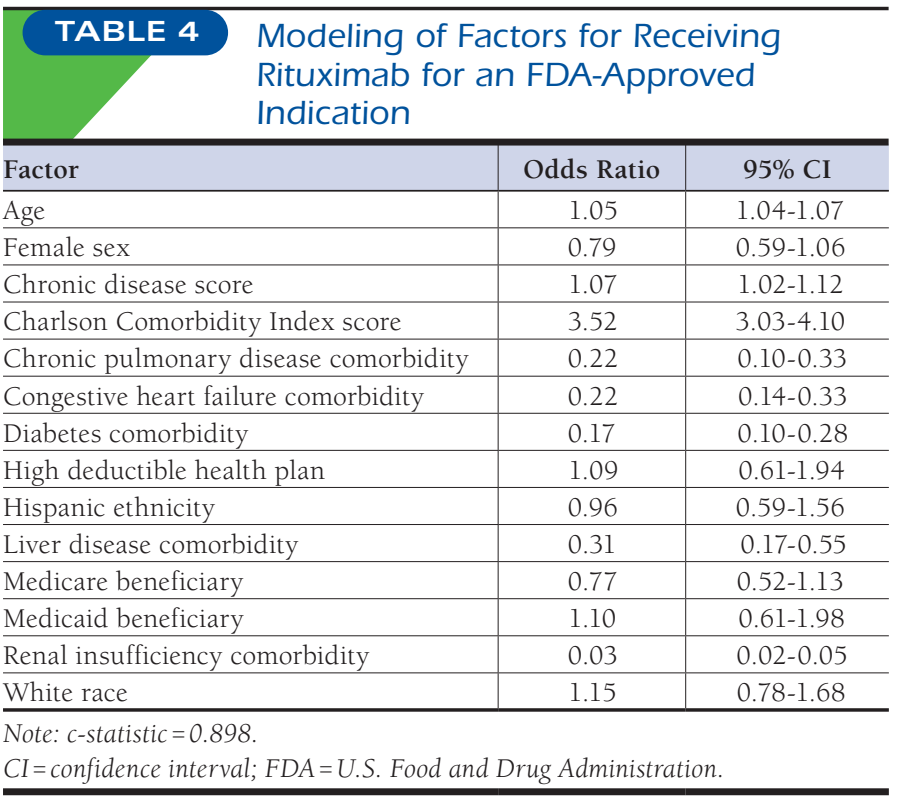

infusions to identify indication. While it is unlikely that subsequent infusions could have been for another indication, this possibility cannot be ruled out.

\section{Conclusions}

While rituximab, an anti-CD20 monoclonal antibody with FDA approval for orphan and nonorphan indications, was used primarily for approved indications, the trend for use in off-label indications dramatically increased over time. With the recent FDA approval of the rituximab biosimilar and its expected lower price, off-label use could continue to rise. Policymakers should explore opportunities to manage this specialty medication with off-label use based on evidence of efficacy/effectiveness.

\section{Authors}

THOMAS DELATE, PhD, MS, Drug Use Management, Kaiser Permanente National Pharmacy, Aurora, Colorado, and Department of Clinical Pharmacy, Skaggs School of Pharmacy and Pharmaceutical Sciences, University of Colorado Anschutz Medical Campus, Aurora. MARGARET L. HANSEN, PharmD, Neurology Department, Wexner Medical Center, The Ohio State University, Columbus; AMARYLIS C. GUTIERREZ, PharmD, California Shared Services, Kaiser Permanente National Pharmacy, Downey, California, and KIM N. LE, PharmD, Drug Use Management, Kaiser Permanente National Pharmacy, Downey, California.

AUTHOR CORRESPONDENCE: Thomas Delate, PhD, MS, Kaiser Permanente National Pharmacy, 16601 E. Centretech Pkwy., Aurora, CO 80011. Tel.: 303.739.3538; E-mail: tom.delate@kp.org.

\section{DISCLOSURES}

This study was funded by Kaiser Permanente. All authors except Hansen are employed by Kaiser Permanente. Hansen has nothing to disclose.

Preliminary results were presented at the Mountain States Conference for Residents and Preceptors in May 2019 in Salt Lake City, UT, and at an encore presentation October 2019 at the American College of Clinical Pharmacy Annual Meeting in New York, NY.

\section{REFERENCES}

1. U.S. Food and Drug Administration. Orphan Drug Act - relevant excerpts. 2013. Available at: https://www.fda.gov/industry/designatingorphan-product-drugs-and-biological-products/orphan-drug-act-relevantexcerpts. Accessed May 12, 2020.

2. Haffner ME. Adopting orphan drugs-two dozen years of treating rare diseases. N Engl J Med. 2006;354(5):445-47.

3. Seoane-Vazquez E, Rodriguez-Monguio R, Szeinbach SL, Visaria J. Incentives for orphan drug research and development in the United States. Orphanet J Rare Dis. 2008;3:33

4. U.S. Food and Drug Administration. Designating an orphan product: drugs and biological products. 2017. Available at: https://www.fda.gov/ industry/developing-products-rare-diseases-conditions/designating-orphanproduct-drugs-and-biological-products. Accessed May 12, 2020.

5. EvaluatePharma. Orphan drug report 2018. May 2018. Available at: https://www.evaluate.com/sites/default/files/media/download-files/OD18 pdf. Accessed May 12, 2020.

6. Kesselheim AS, Myers JA, Solomon DH, Winkelmayer WC, Levin R, Avorn J. The prevalence and cost of unapproved uses of top selling orphan drugs. PLoS One. 2012;7:e31894

7. Rituximab (rituximab injection), solution. Genentech. 2019. Available at: https://medlibrary.org/lib/rx/meds/rituxan/. Accessed May 12, 2020.

8. Kocs D, Fendrick AM. Effect of off-label use of oncology drugs on pharmaceutical costs: the rituximab experience. Am J Manag Care. 2003;9(5):393-400

9. O'Connor K, Liddle C. Prospective data collection of off-label use of rituximab in Australian public hospitals. Intern Med J. 2013;43(8):863-70

10. Van Allen EM, Miyake T, Gunn N, Behler CM, Kohlwes J. Off-label use of rituximab in a multipayer insurance system. J Oncol Pract. 2011;7(2):76-79.

11. Clark DO, Von KM, Saunders K, Baluch WM, Simon GE. A chronic disease score with empirically derived weights. Med Care. 1995;33(8):783-95.

12. Quan H, Sundararajan V, Halfon P, et al. Coding algorithms for defining comorbidities in ICD-9-CM and ICD-10 administrative data. Med Care. 2005;43(11):1130-39.

13. Giuliani J, Bonetti A. The economic impact of biosimilars in oncology and hematology: the case of trastuzumab and rituximab. Anticancer Res. 2019;39(7):3971-73

14. Jurczak W, Długosz Danecka M, Buske C. Rituximab biosimilars for lymphoma in Europe. Expert Opin Biol Ther. 2019;19(10):1045-56.

15. America's Health Insurance Plans. Orphan drug utilization and price changes (2012-2014). Data brief. October 2016. Available at: https://www. ahip.org/wp-content/uploads/2016/10/OrphanDrug_DataBrief_10.21.16.pdf. Accessed May 12, 2020

16. Danés I, Agustí A, Vallano C, et al. Outcomes of off-label drug uses in hospitals: a multicentric prospective study. Eur J Clin Pharmacol. 2014:70(11):1385-93.

17. Berntsson SG, Kristoffersson A, Boström I, Feresiadou A, Burman J, Landtblom AM. Rapidly increasing off-label use of rituximab in multiple sclerosis in Sweden - outlier or predecessor? Acta Neurol Scand. 2018;138(4):327-31 
18. Bar-Or A, Calabresi PA, Arnold D, et al. Rituximab in relapsing-remitting multiple sclerosis: a 72-week, open-label, phase I trial. Ann Neurol. 2008;63(3):395-400.

19. Hauser SL, Waubant E, Arnold DL, et al. B-cell depletion with rituximab in relapsing-remitting multiple sclerosis. N Engl J Med. 2008;358(7):676-88.

20. Hawker K, O'Connor P, Freedman MS, et al. Rituximab in patients with primary progressive multiple sclerosis: results of a randomized double-blind placebo-controlled multicenter trial. Ann Neurol. 2009;66(4):460-71.
21. Naismith RT, Piccio L, Lyons JA, et al. Rituximab add-on therapy for breakthrough relapsing multiple sclerosis: a 52-week phase II trial. Neurology. 2010;74(23):1860-67.

22. Dooms M, Cassiman D, Simoens S. Off-label use of orphan medicinal products: a Belgian qualitative study. Orphanet J Rare Dis. 2016;28;11(1):144. 